\title{
PAPER
}

\section{Pyramidal tract mapping by diffusion tensor magnetic resonance imaging in multiple sclerosis: improving correlations with disability}

\author{
M Wilson, C R Tench, P S Morgan, L D Blumhardt
}

See end of article for authors' affiliations

......................

Correspondence to: Dr Martin Wilson, Department of Neurology, Hope Hospital, Stott Lane, Manchester M6 8HD, UK; doctormartin.wilson@virgin.net

Received 15 April 2002

In revised form

23 October 2002

Accepted

1 November 2002
Background: Current magnetic resonance imaging (MRI) outcome measures such as T2 lesion load correlate poorly with disability in multiple sclerosis. Diffusion tensor imaging (DTI) of the brain can provide unique information regarding the orientation and integrity of white matter tracts in vivo.

Objective: To use this information to map the pyramidal tracts of patients with multiple sclerosis, investigate the relation between burden of disease in the tracts and disability, and compare this with more global magnetic resonance estimates of disease burden.

Methods: 25 patients with relapsing-remitting multiple sclerosis and 17 healthy volunteers were studied with DTI. An algorithm was used that automatically produced anatomically plausible maps of white matter tracts. The integrity of the pyramidal tracts was assessed using relative anisotropy and a novel measure $\left(L_{t}\right)$ derived from the compounded relative anisotropy along the tracts. The methods were compared with both traditional and more recent techniques for measuring disease burden in multiple sclerosis (T2 lesion load and "whole brain" diffusion histograms).

Results: Relative anisotropy and $L_{t}$ were significantly lower in patients than controls $(p<0.05)$. Pyramidal tract $L_{t}$ in the patients correlated significantly with both expanded disability status scale $(r=-0.48, p<0.05)$, and to a greater degree, the pyramidal Kurtzke functional system score (KFS-p) $(r=-0.75, p<0.0001)$. T2 lesion load and diffusion histogram parameters did not correlate with disability.

Conclusions: Tract mapping using DTI is feasible and may increase the specificity of MRI in multiple sclerosis by matching appropriate tracts with specific clinical scoring systems. These techniques may be applicable to a wide range of neurological conditions.
$\mathrm{T}$ he development of magnetic resonance imaging (MRI) beyond a diagnostic aid in multiple sclerosis has been limited by consistently weak relations between MRI measures and the level of current or future disability (the "clinico-radiological paradox"). This in part reflects poor pathological specificity of T2 weighted images, which are unable to differentiate between oedema, demyelination, axonal loss, and gliosis, ${ }^{1-3}$ and the limitations of the expanded disability status scale (EDSS), ${ }^{4}$ which is heavily weighted toward ambulatory dysfunction. The other explanation is related to the concept of "clinically eloquent" sites of involvement in multiple sclerosis, whereby a small lesion in the spinal cord, pyramidal tract, or optic nerve may cause significant impairment, whereas a large subcortical lesion may be entirely asymptomatic. A "burden of disease" measure-such as total T2 lesion volume, for example-will include many asymptomatic lesions and provides no information about the spatial distribution or functional relevance of pathological damage. In addition, such measures do not take into account the pathology within apparently normal appearing white matter.

Various MRI studies that have been applied to these problems include quantitative techniques such as magnetisation transfer imaging, ${ }^{5} \mathrm{Tl}$ relaxometry, ${ }^{6}$ magnetic resonance spectroscopy, ${ }^{7-9}$ and diffusion weighted imaging. ${ }^{10}$ Diffusion weighted imaging uses the random movement of water molecules in tissue to generate image contrast, allowing qualitative assessment of various pathologies including stroke and cerebral tumours. ${ }^{11}$ Diffusion imaging can also provide a quantitative measure of water diffusion in tissue-the apparent diffusion coefficient (ADC). If the ADC is measured along three (or more) appropriate vectors, an average $\mathrm{ADC}\left(\mathrm{ADC}_{\mathrm{av}}\right)$ can be calculated, to provide a quantitative measure of the overall diffusivity of a region. Diffusion in the white matter tracts of the brain is primarily restricted by intact myelinated axons; damage to these structures is presumed to allow less restricted diffusion and raised ADC values. Measurement of ADC within multiple sclerosis plaques has revealed the heterogeneous nature of "T2 lesions" in vivo, ${ }^{12-17}$ and the distribution of ADC throughout the brain has also been used to assess the burden of disease using the technique of whole brain histograms, ${ }^{18-20}$ which include both discrete lesions and normal appearing white matter.

Diffusion tensor imaging (DTI) requires that diffusion gradients be applied in at least six non-colinear directions. This enables the construction of a tensor, a mathematical matrix that describes diffusion within each voxel independently of the orientation of the applied gradients. ${ }^{21}$ The tensor can be used to produce images of both trace (equivalent to ADC averaged between three or more orthogonal directions) and anisotropy (highly anisotropic tissues such as normal white matter producing high signal). However, the directional information contained in the tensor also makes it possible to identify the orientation and integrity of white matter tracts. Preliminary studies in rats ${ }^{22}{ }^{23}$ and subsequently healthy human volunteer ${ }^{24-26}$ have shown the potential of these techniques, and case reports have suggested possible clinical applications in stroke ${ }^{27}$ and neuro-oncology. ${ }^{28}$

Abbreviations: $A D C$, apparent diffusion coefficient; DTI, diffusion tensor imaging; EDSS, expanded disability status scale; KFS-p, Kurtzke functional systems score; ROI, region of interest; TE, time of echo; TR, time of repetition 


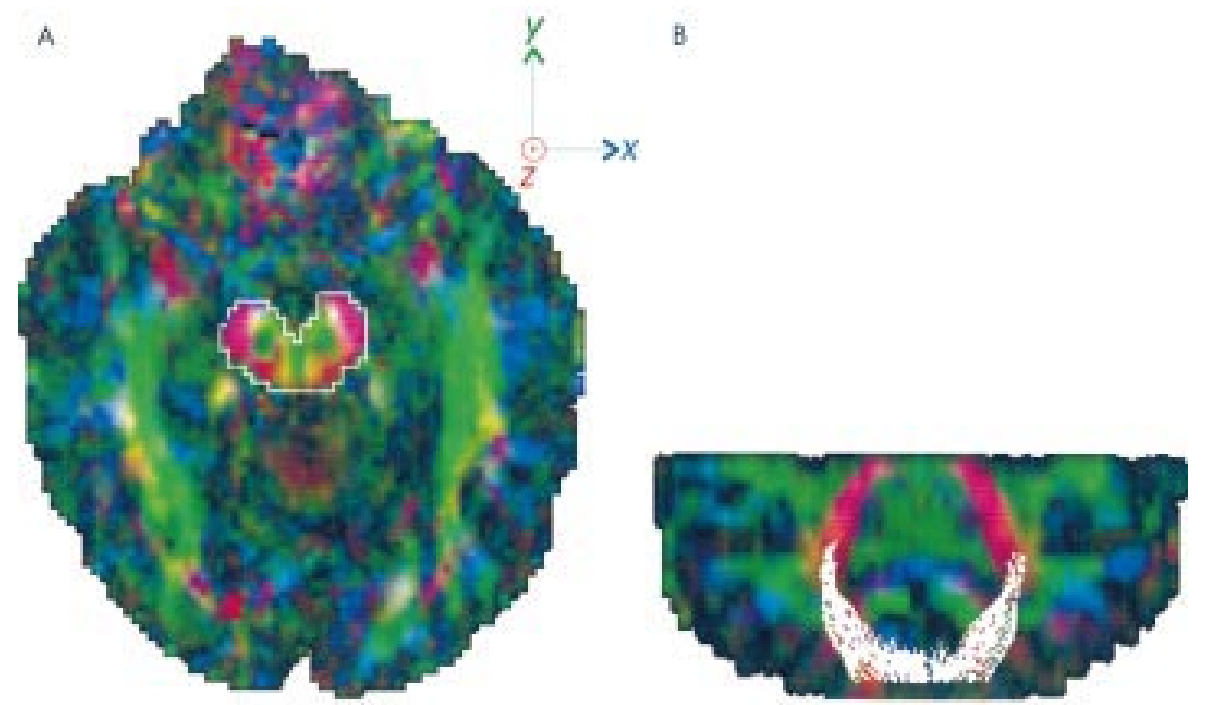

Figure 1 Pyramidal tract mapping begins with the definition of a region of interest (in this case the midbrain) on an axial image $(A)$ in which the orientations of fibres in $x, y$, and $z$ planes are colour coded blue, green, and red, respectively. Trajectories are then mapped automatically by making single steps in the direction of the principal diffusion direction. The resulting tract map is shown in coronal section in (B).

Using DTI, we aimed to assess pyramidal tract damage caused by multiple sclerosis by using an algorithm to both map the tracts and measure the burden of disease within them. We hypothesised that, by studying specific anatomical regions relevant to motor impairment, the correlation between MRI parameters and the level of clinical disability would be significantly improved in comparison with either total T2 lesion volume or whole brain diffusion histograms.

\section{METHODS}

We studied 25 patients with relapsing-remitting multiple sclerosis and 17 healthy volunteers. Informed consent from all subjects was obtained in accordance with the Declaration of Helsinki and with approval of the local ethics committee.

Each patient underwent neurological assessment including rating on the EDSS and the pyramidal component of the Kurtzke functional systems score (KFS-p). The KFS-p was used as a more specific estimate of motor impairment. There were eight male and 17 female patients. Their mean age was 40 years (range 26 to 54), mean disease duration seven years (range 1 to 21 ), and mean EDSS score 3 (range 1 to 6).

The mean age of the control subjects was 37 years (range 25 to 49$)$.

No patient had been exposed to disease modifying drugs at any time, or received steroids in the three months before the study. All image analysis was performed blind to the identity of the subject.

\section{MRI protocols}

All subjects were scanned in a clinical imaging system at 1.5T (Magnetom Vision, Siemens, Erlangen, Germany). A spin echo, echo planar diffusion weighted sequence was used (TE $103 \mathrm{~ms}$, TR $6000 \mathrm{~ms}$, time per echo $0.8 \mathrm{~ms}$, number of echoes 96 , voxel dimensions $2 \times 2 \times 4 \mathrm{~mm}$ ) with the application of six non-colinear gradients (maximum strength $22 \mathrm{mT} / \mathrm{m}$ ) with a $\mathrm{b}_{\max }$ of $1000 \mathrm{~s} / \mathrm{mm}^{2}{ }^{29}$ Fifteen axial slices were imaged, sampling a volume that encompassed the lateral ventricles and included the cerebral peduncles and midbrain in the most caudal slices. Acquisition was preceded by an automated shimming sequence to maximise magnetic field homogeneity. The tensor data were transferred electronically to a workstation (Sun Microsystems Ultra 1) for processing of trace and relative anisotropy maps, using locally developed software based on definitions published by Basser and Pierpaoli. ${ }^{21}$ This software included a distortion correction using the "reversed gradient method," which requires the acquisition of two datasets, the second with a phase encoding gradient of reversed polarity. ${ }^{29}$ Post-processing took approximately three minutes per image set.

In addition, all subjects were scanned using a standard T2 weighted dual echo sequence $(\mathrm{TE}=80 \mathrm{~ms}$, $\mathrm{TR}=2323 \mathrm{~ms}$, voxel dimensions $1 \times 1 \times 3 \mathrm{~mm})$.

\section{T2 lesion load}

The total volume of brain lesions on T2 weighted spin echo images was estimated using a semiautomated thresholding technique within a commercial image analysis software program (Analyze ${ }^{\circ}$, Analyzedirect.com, USA).

\section{Diffusion histograms}

"Whole brain" diffusion histograms were calculated within the Analyze $\odot$ program using methods published previously. ${ }^{20}$ Histograms of both trace diffusion and relative anisotropy were calculated and normalised to total brain volume, from which histogram parameters including mean, standard deviation, and quartile values could be derived.

\section{Pyramidal tract mapping}

In normal white matter the direction of highest diffusivity, the principal diffusion vector, is parallel to the tract. ${ }^{30-32}$ Using this fact, algorithms have been developed which map specific white matter tract bundles automatically, by following a trajectory based upon the principle diffusion vector in each adjacent voxel. ${ }^{23} 2426$

To initialise a trajectory, the start point is taken from within a region of interest (ROI), which is used to mark out a specific part of the brain on the acquired image. In the present study a region of interest in the midbrain, which included the pyramidal tracts, was defined using a semiautomated thresholding technique (fig 1). The pyramidal tracts were chosen because they are highly anisotropic, have a well defined anatomical course, and are clinically relevant to motor impairment.

To prevent initial points being chosen from outside the white matter, the relative anisotropy at the initial point must be greater than a predefined threshold value. The relative anisotropy measures the relative differences between the three principal diffusivities and is highest in white matter. A threshold value of 0.1 was used to reject any points of grey matter within the region of interest. 


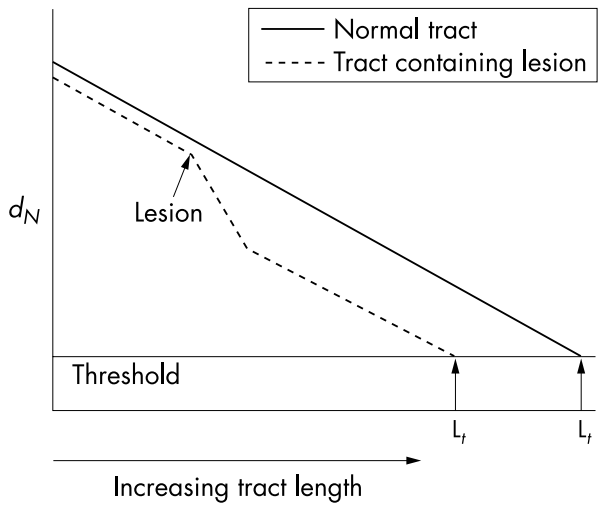

Figure 2 Schematic representation of the measurement of $L_{\text {t }}$ defined as the point at which $d_{N}$ falls below a threshold value. The solid line represents a trajectory from a normal control, the dashed line a trajectory passing through a region of low anisotropy (lesion). Note that although the trajectory is able to continue through the lesion, this results in a reduction of trajectory length $L_{\text {. }}$.

From the initial point, the trajectory is continued until a predefined stopping criterion is met. The stopping criteria in this study were chosen carefully so that trajectories could pass through a multiple sclerosis lesion but not enter the grey matter. Here a parameter based on the relative anisotropy is used to determine the termination point of the trajectory and therefore its length $\mathrm{L}_{t}$. In normal appearing white matter of patients, and in lesions, the relative anisotropy is reduced compared with that of white matter in healthy controls. This fact can be used to reduce the length of trajectories that follow tracts with a high disease burden. A simple measure of burden up to the $N^{\text {th }}$ step along the tract is the compounded relative anisotropy at each point $r_{n}$ as defined by:

$d_{N}=\prod_{n=1}^{n} \mathrm{RA}\left(\mathbf{r}_{n}\right)$

With this measure, the disease burden is represented equally at all points in the tract. Termination of the trajectory occurs when $d_{N}$ falls below a threshold value which is set so as to prevent the trajectory entering the grey matter. The trajectory length $\left(\mathrm{L}_{t}\right)$ at which this threshold is crossed is therefore a measure of pathological damage along the tract (fig 2).

\section{Statistics}

Differences in the diffusion characteristics of the tract maps and histograms in patients and controls were compared with the Mann-Whitney test. Parameters from the tract maps and histograms were correlated with both global disability (EDSS) and motor impairment (KFS-p) using Spearman's rank method. Two sided probability $(\mathrm{p})$ values and a significance level of $<0.05$ were used throughout.

\section{Reproducibility}

The algorithm for identifying and mapping tracts was fully automated, but required a user defined region of interest from which to start. Despite being blinded to the subject identity this manual input could potentially introduce bias and error. In order to assess the error that this manual step might introduce, ROI definition was repeated by another investigator who was unaware of the previous ROI position. This was done on all 25 patients, and pyramidal tract data were collected and analysed as previously described. Parameters were then compared and the coefficient of error calculated. ${ }^{33}$
Table 1 Comparison of pyramida tract trajectory length $\left(L_{t}\right)$ in patients and controls

\begin{tabular}{lll}
\hline & $\begin{array}{l}\text { Controls } \\
(\mathbf{n}=17)\end{array}$ & Patients $(\mathbf{n}=25)$ \\
\hline Mean $\mathrm{L}_{t}(\mathrm{~mm})$ & 21.40 & $19.99(\mathrm{p}<0.05)$ \\
SD L $(\mathrm{mm})$ & 10.95 & $9.56(\mathrm{p}<0.05)$ \\
Q75 $\mathrm{L}_{t}(\mathrm{~mm})$ & 29.5 & $25.5(\mathrm{p}<0.05)$ \\
\hline
\end{tabular}

Mean values from each subject group are shown, with corresponding $p$ values (Mann-Whitney).

Table 2 Spearmans' correlation coefficients $(r)$ for pyramidal tract trajectory length $\left(L_{t}\right)$ values with overall (EDSS) and specific (KFS-p) disability scores

\begin{tabular}{lll}
\hline & EDSS & KFS-p \\
\hline Mean $\mathrm{L}_{t}$ & $r=-0.36, \mathrm{p}=0.07$ & $r=-0.63, \mathrm{p}=0.001$ \\
SD $\mathrm{L}_{t}$ & $r=-0.48, \mathrm{p}<0.05$ & $r=-0.48, \mathrm{p}<0.01$ \\
$\mathrm{Q}_{75} \mathrm{~L}_{t}$ & $r=-0.49, \mathrm{p}<0.05$ & $r=-0.75, \mathrm{p}<0.0001$ \\
\hline
\end{tabular}

EDSS, expanded disability status scale; KFS-p, Kurtzke functional systems score; $Q_{75}, 75$ th quartile.

\section{RESULTS}

\section{Pyramidal tract data}

Interobserver coefficient of error for all pyramidal tract parameters was $<1 \%$. The mean, standard deviation, and 75th quartile $\mathrm{L}_{t}$, measured along the pyramidal tracts, were significantly lower in patients than controls (all p < 0.05) (table 1). Means and standard deviations of relative anisotropy were also significantly lower in the pyramidal tracts of the patients $(\mathrm{p}<0.05)$.

There were strong correlations between pyramidal tract $\mathrm{L}_{t}$ and the KFS-p (measuring "pyramidal" impairment), particularly for the 75 th quartile $\mathrm{L}_{t}$ (table 2 ), reflecting a reduction of trajectories with high $\mathrm{L}_{t}$. The relation with EDSS (measuring overall disability) was not as strong, though the $\mathrm{L}_{t}$ standard deviation and 75th quartile remained significant.

The mean relative anisotropy of the pyramidal tracts also showed significant correlations with KFS-p $(r=-0.69$, $\mathrm{p}<0.001)$, though not with the EDSS $(r=-0.36, \mathrm{p}=0.08)$.

\section{Diffusion histogram data}

The trace histograms revealed significant differences between patients and controls, particularly in peak height of the histogram, which was lower in patients $(p<0.05)$ with a consequent right shift toward higher values of trace (less restricted diffusion) (fig 3). However, peak height and mean trace did not correlate significantly with disease duration, EDSS, or KFS-p. Histograms of relative anisotropy revealed more subtle differences in patients compared with controls, with a left shift toward lower values of anisotropy (fig 4). These differences did not reach statistical significance, however. Mean and peak height relative anisotropy did not correlate with EDSS or KFS-p.

\section{T2 lesion volume}

The total volume of T2 lesions (mean $5482 \mathrm{~mm}^{3}$, range 4 to $47590 \mathrm{~mm}^{3}$ ) did not correlate significantly with EDSS $(r=-0.13, \mathrm{p}=0.53)$, or KFS-p $(r=0.11, \mathrm{p}=0.6)$.

\section{DISCUSSION}

In this paper we describe a technique based on diffusion tensor imaging that automatically maps white matter tracts in specific regions of interest (for example, the pyramidal tracts) and measures diffusion characteristics along these tracts. We have demonstrated significant differences between multiple 


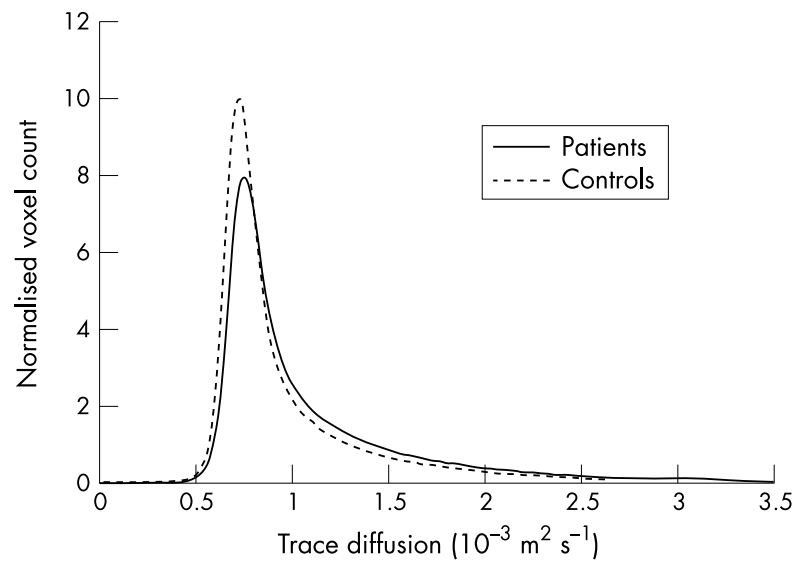

Figure 3 Normalised histograms of trace diffusion in patients and controls. The histogram of multiple sclerosis patients shows a lower peak height and a right shift toward higher, less restricted diffusion.

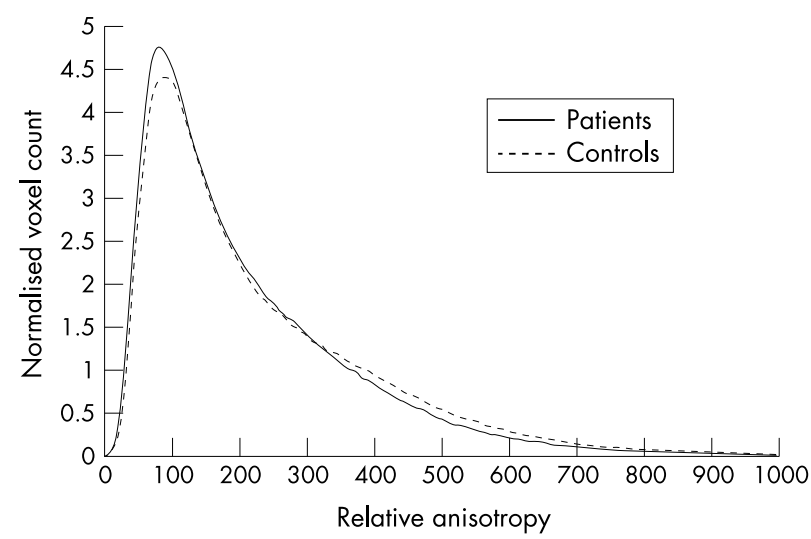

Figure 4 Normalised histograms of relative anisotropy in patients and controls. The histogram of multiple sclerosis patients shows a higher peak and left shift toward lower values of anisotropy, implying loss of normal white matter fibre organisation.

sclerosis patients and healthy controls, and significant correlations between pyramidal tract abnormalities and levels of clinical impairment.

Other investigators have also used DTI to identify white matter tracts in human subjects. In their study of healthy volunteers, for example, Basser et al used an algorithm with stopping criteria based on a minimum allowed relative anisotropy threshold. ${ }^{26}$ However, this raises problems when applied to pathological conditions such as multiple sclerosis, where lesions would be expected to cause low values of anisotropy and would therefore be excluded from any analysis along the identified tracts.

The algorithm described in this paper produces anatomically plausible fibre tract maps, allowing the identification of specific regions of interest (in this case, the pyramidal tracts). Having identified the target tracts, diffusion characteristics within them can then be measured. In this study we have used a length measure $\left(\mathrm{L}_{t}\right)$, based on the relative anisotropy in the tract bundles, that allows the accumulation of data through both healthy and diseased tissue. This measure provides an unbiased representation of disease burden at all points along the tract.

We have shown that these parameters are significantly different in multiple sclerosis patients and age matched healthy controls, and correlate with the degree of impairment and disability, particularly the pyramidal component of the EDSS. This shows the potential of the technique to match abnormalities in appropriate anatomical fibre tracts to specific disability measures, and improve the specificity of MRI for the pathological substrates of disability in multiple sclerosis. Other investigators have also attempted to improve correlations with clinical disability by measuring T2 lesion volume within the pyramidal tracts from standard T2 weighted MRI ${ }^{34}$ Although this resulted in significant correlations with EDSS $(r=0.67)$, they also found an unusually high correlation between total brain T2 lesion load and EDSS $(r=0.6)$ in their patient group, making any advantage gained minimal. We did not feel that measuring lesion volume within such a small region was practical using the techniques at our disposal.

In contrast to the pyramidal tract data, the other measures of disease burden we assessed failed to provide any significant correlations with disability. These included both total T2 lesion volume and diffusion histogram analysis (which allows a more complete assessment of disease burden by including pathology within normal appearing white matter as well as discrete lesions). It seems likely that this lack of correlation with disability reflects the heavy weighting of the EDSS toward motor impairment and its relative insensitivity to other domains including cognitive function. The results of our study, therefore, suggest that it is the site of pathology rather than the overall burden of disease that is most relevant to disability (as defined by the EDSS), and that the clinicoradiological paradox may be improved by matching disability measures to anatomically appropriate MRI measures.

The methods described have potential applications in many branches of neuroscience, though some limitations should be mentioned. The technique is dependent on image resolution, so mapping tract bundles of subvoxel size would not be possible. In addition, fibre tracts with lower anisotropy and less well defined anatomy than the pyramidal tracts may be more difficult to track. Extending image acquisition to include the brain stem and posterior fossa presents technical difficulties in producing artefact-free images and would increase acquisition time.

The identified tracts are not restricted to measuring diffusion characteristics, however. By co-registering tensor images with other modes, a whole range of magnetic resonance parameters within specific tracts could be measured, including spectroscopy, Tl relaxation times, and magnetisation transfer ratios. As such we believe the methods described here may be applied to a variety of neurological conditions including multiple sclerosis, stroke, cerebral tumours, dementia, and psychiatric disorders.

Authors' affiliations

M Wilson, C R Tench, L D Blumhardt, Division of Clinical Neurology, Queens Medical Centre, Nottingham University, Nottingham, UK P S Morgan, Division of Academic Radiology, Nottingham University

Competing interests: none declared

\section{REFERENCES}

1 Miller DH, Grossman RI, Reingold SC, et al. The role of magnetic resonance techniques in understanding and managing multiple sclerosis. Brain 1998;121:3-24.

2 Stevenson VL, Miller DH. Magnetic resonance imaging in the monitoring of disease progression in multiple sclerosis. Multiple Sclerosis 1999;5:268-72

3 van Waesberghe JH, Kamphorst W, De Groot CJ, et al. Axonal loss in multiple sclerosis lesions: magnetic resonance imaging insights into substrates of disability. Ann Neurol 1999;46:747-54.

4 Kurtzke JF. Rating neurologic impairment in multiple sclerosis: an expanded disability status scale (EDSS). Neurology 1983;33:1444-52.

5 Grossman RI, Gomori JM, Ramer KN, et al. Magnetization transfer: theory and clinical applications in neuroradiology. Radiographics 1994; 14:279-90.

6 Barbosa S, Blumhardt LD, Roberts N, et al. Magnetic resonance relaxation time mapping in multiple sclerosis: normal appearing white matter and the "invisible" lesion load. Magn Reson Imaging $1994 ; 12: 33-42$. 
7 Davie CA, Hawkins CP, Barker GJ, et al. Serial proton magnetic resonance spectroscopy in acute multiple sclerosis lesions. Brain 1994; 117:49-58.

8 Davie CA, Barker GJ, Webb S, et al. Persistent functional deficit in multiple sclerosis and autosomal dominant cerebellar ataxia is associated with axon loss. Brain 1995:118:1583-92.

9 Gonen O, Catalaa I, Babb JS, et al. Total brain N-acetylaspartate: a new measure of disease load in MS. Neurology 2000;54:15-19.

10 Horsfield MA, Larsson HB, Jones DK, et al. Diffusion magnetic resonance imaging in multiple sclerosis. J Neurol Neurosurg Psychiatry 1998;64(suppl 1):S80-4.

11 Rowley HA, Grant PE, Roberts TP. Diffusion MR imaging. Theory and applications. Neuroimaging Clin North Am 1999;9:343-61.

12 Horsfield MA, Lai M, Webb SL, et al. Apparent diffusion coefficients in benign and secondary progressive multiple sclerosis by nuclear magnetic resonance. Magn Reson Med 1996;36:393-400.

13 Filippi $M$, lannucci $G$, Cercignani $M$, et al. A quantitative study of water diffusion in multiple sclerosis lesions and normal-appearing white matter using echo-planar imaging. Arch Neurol 2000;57:1017-21.

14 Christiansen P, Gideon P, Thomsen $\mathrm{C}$, et al. Increased water self-diffusion in chronic plaques and in apparently normal white matter in patients with multiple sclerosis. Acta Neurol Scand 1993;87: 195-9.

15 Roychowdhury S, Maldjian JA, Grossman RI. Multiple sclerosis: comparison of trace apparent diffusion coefficients with MR enhancement pattern of lesions. Am J Neuroradiol 2000;21:869-74.

16 Tievsky AL, Ptak T, Farkas J. Investigation of apparent diffusion coefficient and diffusion tensor anisotrophy in acute and chronic multiple sclerosis lesions. Am J Neuroradiol 1999;20:1491-9.

17 Werring DJ, Clark CA, Barker GJ, et al. Diffusion tensor imaging of lesions and normal-appearing white matter in multiple sclerosis. Neurology 1999;52:1626-32

18 Cercignani $M$, lannucci G, Rocca MA, et al. Pathologic damage in MS assessed by diffusion-weighted and magnetization transfer MRI. Neurology 2000;54:1139-44.

19 Nusbaum AO, Tang CY, Wei T, et al. Whole-brain diffusion MR histograms differ between MS subtypes. Neurology 2000;54:1421-7.

20 Wilson M, Morgan PS, Lin X, et al. Quantitative diffusion weighted magnetic resonance imaging, cerebral atrophy, and disability in multiple sclerosis. J Neurol Neurosurg Psychiatry 2001;70:318-22.

21 Basser PJ, Pierpaoli C. A simplified method to measure the diffusion tensor from seven MR images. Magn Reson Med 1998;39:928-34.
22 Mori S, Crain BJ, Chacko VP, et al. Three-dimensional tracking of axonal projections in the brain by magnetic resonance imaging. Ann Neurol 1999;45:265-9.

23 Xue $\mathbf{R}$, van Ziil PC, Crain BJ, et al. In vivo three-dimensional reconstruction of rat brain axonal projections by diffusion tensor imaging Magn Reson Med 1999;42:1123-7.

24 Tench C, Wilson M, Morgan $\mathrm{P}$, et al. White matter fibre tractography in multiple sclerosis using diffusion tensor imaging. Proc Int Soc Magn Res 2001;2:1420.

25 Jones DK, Simmons A, Williams SC, et al. Non-invasive assessment of axonal fiber connectivity in the human brain via diffusion tensor MRI. Magn Reson Med 1999;42:37-41

26 Basser PJ, Pajevic S, Pierpaoli C, et al. In vivo fiber tractography using DT-MRI data. Magn Reson Med 2000;44:625-32.

27 Werring DJ, Toosy AT, Clark CA, et al. Diffusion tensor imaging can detect and quantify corticospinal tract degeneration after stroke. J Neurol Neurosurg Psychiatry 2000;69:269-72

28 Wieshmann UC, Symms MR, Parker GJ, et al. Diffusion tensor imaging demonstrates deviation of fibres in normal appearing white matter adjacent to a brain tumour. J Neurol Neurosurg Psychiatry 2000;68:501-3.

29 Morgan PS, Moody AR, Allder SJ, et al. Correction of distortion in ADC maps using the reversed gradient method. Proc Int Soc Magn Res Med 2000;8:800

30 Moseley ME, Cohen Y, Kucharczyk J, et al. Diffusion-weighted MR imaging of anisotropic water diffusion in cat central nervous system. Radiology 1990;176:439-45.

31 Scollan DF, Holmes A, Winslow R, et al. Histological validation of myocardial microstructure obtained from diffusion tensor magnetic resonance imaging. Am J Physiol 1998;275:H2308-18.

32 Hsu EW, Muzikant AL, Matulevicius SA, et al. Magnetic resonance myocardial fiber-orientation mapping with direct histological correlation. Am J Physiol 1998;274:H1627-34.

33 Bland JM. An introduction to medical statistics. Oxford: Oxford University Press, 1991.

34 Riahi F, Zijdenbos A, Narayanan S, et al. Improved correlation between scores on the expanded disability status scale and cerebral lesion load in relapsing-remitting multiple sclerosis. Results of the application of new imaging methods. Brain 1998;121:1305-12.

\title{
NEUROLOGICAL STAMP
}

\author{
Georges Marinescu (1864-1938)
}

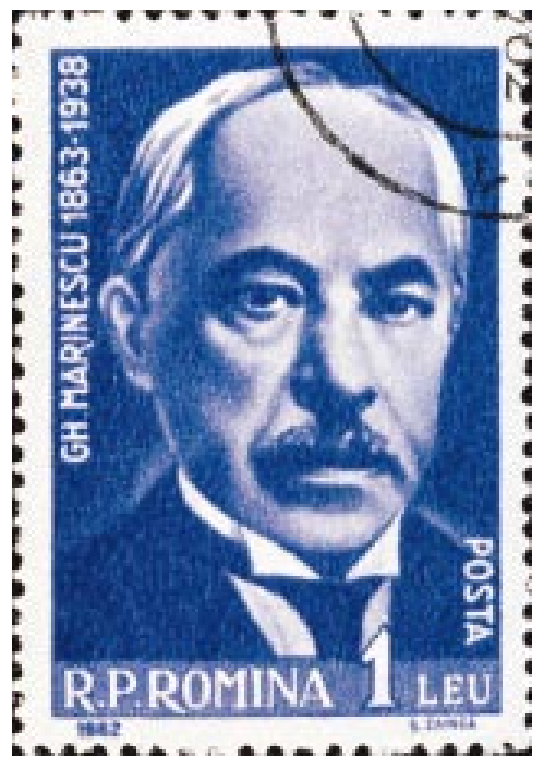

arinescu was Romanian and a pupil of Charcot, who became professor of neurology in Bucharest. He contributed knowledge to the anatomy and pathology of the nervous and observed the fatal effects of removal of the pituitary gland in animals. Alzheimer described neurofibrillary tangles and sclerotic plaques found in Alzheimer's disease in 1906 and 1907. Earlier in 1892, Georges Marinescu with Paul Blocq had modified the staining techniques for glia (previously developed by Santiago Ramon y Cajal) and observed plaques in the cortices of two senile individuals with gross memory defects, mental confusion, aphasia, and apraxia. With Nicolesco he reported on the clinical aspects of thalamic lesions, and elaborated further on what was already known about the thalamic syndrome. With Bloce, in 1893 he reported case of Parkinson's disease associated with a tuberculoma in the substantia nigra. These observations were the basis of Edouard Brissaud's suggestion in 1894 of the nigral origin of this disorder. Eponymically Marinescu is remembered for the Marinescu-Sjogren syndrome (cataracts, mental retardation, short stature delayed sexual development, and ataxia). Marinescu was honoured philatelically by a stamp issued by Romania in 1962 (Stanley Gibbons no 2943, Scott no 1496).

L F Haas 